\title{
Linx
}

LinX Revue des linguistes de l'université Paris X Nanterre

59 | 2008

Les conjonctions en diachronie : parcours sémantiques

\section{Sur la genèse des locutions participiales}

\section{Thomas Verjans}

\section{(2) OpenEdition}

12 Journals

Édition électronique

URL : http://journals.openedition.org/linx/624

DOI : $10.4000 / \operatorname{linx} .624$

ISSN : 2118-9692

Éditeur

Presses universitaires de Paris Nanterre

\section{Édition imprimée}

Date de publication : 1 décembre 2008

Pagination : 47-60

ISSN : 0246-8743

\section{Référence électronique}

Thomas Verjans, «Sur la genèse des locutions participiales », Linx [En ligne], 59 | 2008, mis en ligne le 01 janvier 2012, consulté le 06 mai 2019. URL : http://journals.openedition.org/linx/624 ; DOI

$10.4000 /$ linx.624 


\title{
Sur la genèse des locutions participiales
}

\author{
Thomas Verjans \\ Université de Bourgogne - GReLISC (CPTC, EA 4178) \\ E STIH (EA 4089)
}

\section{Introduction}

Les conjonctions de subordination constituent une catégorie remarquable pour son caractère hétérogène aussi bien que pour sa labilité. La diversité des termes qui sont ordinairement regroupés sous ce chef rend délicate non seulement une définition précise de la catégorie ${ }^{1}$, mais plus encore la délimitation d'un ensemble suffisamment cohérent pour donner prise à une étude de nature systématique. Un groupe peut cependant être isolé : le groupe des locutions conjonctives fondées sur une base verbale au mode participe. On peut en dresser, à partir des différents ouvrages de référence, la liste suivante :

\section{Locutions fondées sur une forme en -ant :}

En admettant que, (en) attendant que, en supposant que, moyennant que, nonobstant (ce) que, soidisant que, suivant que, pendant (ce) que, durant (ce) que, considérant que, obstant que, non contrestant que.

Étant donné que, étant admis que.

Maintenant que, cependant que / en cependant que.

\footnotetext{
${ }^{1}$ Catégorie à ce titre souvent critiquée. Voir, par exemple, pour l'une des critiques les plus sévères, P. Le Goffic, selon qui la catégorie est « inutile et parasitaire » $(2001: 42$, n.1).
} 


\section{Locutions fondées sur une forme de participe passé :}

Attendu que, excepté que, hormis que, joint que, supposé que, posé que, pourvu que, vu que, considéré que, entendu que.

Compte tenu que.

Une telle liste appellerait naturellement bon nombre de remarques, concernant notamment l'apparente disparité des unités ainsi réunies, tant sur le plan morphologique de leur structure interne que sur celui des degrés de figement atteint par telles ou telles d'entre elles. Plusieurs arguments pourraient ainsi s'opposer à l'inclusion de l'une ou l'autre de ces locutions. Il n'est néanmoins pas possible d'entreprendre ici l'ensemble de ces discussions, et notre propos se concentrera en conséquence sur la seule question de la genèse de ces locutions, principalement envisagée d'un point de vue morphosyntaxique ${ }^{2}$. Nous nous attacherons plus spécialement ici à quelques-unes de ces locutions, généralement tenues pour exemplaires, en ce sens qu'elles condensent l'ensemble des problèmes posés par cette catégorie (en particulier : durant que, attendu que et moyennant que). Nous verrons alors que les explications généralement invoquées présupposent, en définitive, une conception particulière des locutions conjonctives, qui ne les tient que pour des réalités discursives en posant l'existence d'un régime prépositionnel construit à partir d'un marqueur que et d'un verbe à temps fini. C'est pourquoi, dans le dernier point de cet article, nous proposerons les premiers éléments d'une modélisation alternative, fondée sur un processus analogique en partie inspiré de la théorie cosérienne.

\section{L’évolution des constructions participiales}

La plupart des modifications morphosyntaxiques intervenues dans l'évolution de ces constructions ont été bien répertoriées, mais toujours avec, en ligne de mire, l'acquisition d'un statut prépositionnel par les formes verbales. Trois phénomènes ont été plus particulièrement remarqués : la mobilité du verbe au sein de la construction, la question de l'accord de la forme participiale et, enfin, la réinterprétation des rôles relatifs des constituants.

Le premier phénomène permettant d'établir un lien entre les constructions participiales absolues et les prépositions déverbales tient à l'ordre relatif des constituants au sein du syntagme participial. Or, semble-t-il, dès l'ancien français, dans les constructions mettant en jeu voiant et oiant, se manifeste une tendance à l'antéposition de la forme verbale, au moins lorsque l'agent est de nature nominale. C'est du moins ce que l'on peut constater à la vue des exemples proposés par T. Arnavielle dans le chapitre consacré aux «constructions avec autonomie de l'agent et forme en -ant prédicative (dites «absolues»)» (1997: 237 sq.), où il affirme en outre, dans le cas du

\footnotetext{
2 Pour une étude plus complète de la catégorie ainsi que pour les détails du corpus auquel nous nous référons ici, nous nous permettons de renvoyer à Verjans (2009 : 465-546 \& sous presse). Précisons simplement qu'outre nos lectures personnelles, celui-ci s'appuie sur les bases de données existantes : $B F M, D M F$ et $B H C$, auxquelles s'ajoutent les dictionnaires usuels.
} 
verbe ouir, que, lorsque "l'agent est un nom », il est « le plus souvent postposé » (ibid. : 238). Ainsi, la modification du placement de la forme verbale est-elle présentée, par B. Kortmann (1992) ainsi que E. König et B. Kortmann (1991, 1992), comme l'un des facteurs les plus importants dans le cadre de la réanalyse ${ }^{3}$, en anglais, des participes verbaux en prépositions :

One of the most important changes involved in the categorical reanalysis of verbs is a change in word order. Prepositions precede their complements whereas converbs in absolute constructions, that is, one of the relevant syntactic sources, follow their subjects, with exception for the earliest periods of English. [Kortmann \& König 1992 : 674]

La mobilité des constituants au sein de ces syntagmes a pu s'accompagner de modifications qui ont provoqué une réinterprétation des rôles des constituants. Cela concerne en premier lieu la question de l'accord. Celui-ci se fait en effet en fonction de deux critères. D'abord, en fonction du rôle joué par l'élément nominal :

En français, les principaux cas à examiner sont excepté, réservé, vu, entendu, oü, attendu, compris, passé, supposé, approuvé, qui sont invariables devant le substantif et variables après, ensuite hormis, forme moderne de hors mis et qui maintenant a pris absolument la valeur d'une préposition, et également $c i$ joint, ci-inclus. [Meyer-Lübke 1974 : 176]

Ensuite, l'accord se fait en fonction de la position du verbe au sein du syntagme participial : alors que la postposition du verbe à son agent provoque généralement l'accord, son antéposition, en revanche, et notamment lorsque l'agent est nominal, a tendance à le neutraliser.

As a result of their prenominal position in absolute constructions, participles lost their inflectional endings through which they manifest agreement with their subject, and this loss created a further favorable condition for reanalysis. [Kortmann \& König 1992 : 675]

Il faut toutefois ajouter que, bien que l'accord semble effectué de façon plus régulière dans le cas des formes de participes passés,

la flexion de la forme en ant relève du système des adjectifs dont le thème est commun au masculin et au féminin; l'adjonction d'un - $e$ au féminin est rare et tardive. [Andrieux \& Baumgartner 1983 : 230]

\footnotetext{
${ }^{3}$ Notons que, pour B. Kortmann, et dans ce cas très précis, «both terms (i.e. réanalyse et grammaticalisation) are equally well applicable to the development of verbal forms into prepositions or conjunctions » $(1992: 430)$.
} 
Les corpus que nous avons constitués confirment très largement cette réserve concernant l'accord puisque l'on obtient les résultats suivants ${ }^{4}$ :

Durant: 121 occurrences, 70 accordées, dont 66 au masculin singulier. Moyennant: 28 occurrences, 10 accordées, dont 6 au masculin singulier. Attendu: 4 occurrences, 2 accordées au masculin singulier.

De fait, ce dernier phénomène a également pu jouer en faveur de la réinterprétation du participe comme préposition en lui conférant, au moins en apparence, un caractère invariable lorsque l'accord devait se faire au féminin ${ }^{5}$.

C'est à partir de ces deux phénomènes qu'une réinterprétation des constituants a été rendue possible. En effet, les constructions participiales supposent la présence d'un agent, et celui-ci aurait ainsi précisément été réinterprété en tant que régime de la préposition :

In the reanalysis of absolute constructions (...) as prepositional phrases, the subjects (external arguments) of the relevant verbs are reanalyzed as complements (internal arguments) of prepositions. This process may be supported, on the one hand, by an (oblique) case marking of the argument. (...) On the other hand, reanalysis of a preposed participle as a preposition gains support if the former no longer exhibits agreement with a following argument. In Modern French this is often even obligatorily so, e.g. for attendu ou vu (...). [Kortmann \& König 1991 : 115]

Les prépositions ainsi formées auraient alors pu servir de base au développement des locutions participiales, dont le développement est présenté, par B. Kortmann, comme relevant d'un processus similaire. Justifiant ainsi une étude conjointe des prépositions et des conjonctions, il affirme alors :

There is a considerable proportion of departicipial prepositions (about a quarter of the English and a third of all French instances) which may also take sentential complements (optionnaly introduced by a complementizer), i.e. developed a use as conjunctions (E. considering, barring, notwithstanding, granted, or Fr. durant que, pendant que, hormis que, etc.). In fact, these cases represent about $50 \%$ of all departicipial conjunctions in these two languages. [...] The various factors that are involved in the type of reanalysis

\footnotetext{
${ }^{4}$ Précisons toutefois que nous avons considéré comme accordées par défaut les occurrences au masculin singulier. Il nous semble cependant que ces cas ne peuvent être à proprement parler tenus pour significatifs.

${ }^{5}$ En plus de l'accord en genre et en nombre, auquel nous nous sommes jusqu'à présent limité, il est un autre argument qui peut être invoqué, à tout le moins pour la période de l'ancien français : il s'agit du rôle joué par le cas. Comme le remarque G. Zink : « la construction oiant (veant) toz les barons, où l'on serait tenté de reconnaître une proposition participiale (tous les barons l'entendant / le voyant), doit, en réalité, s'analyser comme un syntagme prépositionnel (en présence de tous les b. : d'où le CR (i.e. le cas régime (tv))). De là sont sorties les prépositions durant, pendant (et la conjonction cependant (...))» [Zink $1993: 67]$.
} 
considered here are valid independently of the complexity of the argument of the underlying non-finite verb form. [Kortmann 1992 : 432-433]

Comme on a pu le voir, les différents phénomènes invoqués conduisent à une grammaticalisation des formes participiales en prépositions. La modélisation suivante, en partie inspirée de Cl. Buridant (2000 : \256), peut résumer l'entier de ce processus, que nous illustrons ici avec le cas de durant:

1) Monstrelet, Chronique, p. 104 : Au regard des délis communs commis ou perpétrés par les subgectz de l'une desdictes parties en obéyssance de l'aultre lesdictes trièves durans, la congnoissance, juridiction et pugnicion en sera et appertendra à la justice du parti où le cas auroit esté commis.

2) Froissart, 1, p. 399 : Les trieuwes durant entre le duch de Normendie et ceuls de Thun l'Evesque, li contes Guillaumes de Hainnau retourna en son pais et vint en la ville de Mons, et la trouva le signeur de Biaumont et de Chimai, son oncle, qui li recorda tout l'estat de son pais et conment on s'i estoit porté depuis son departement.

3) QJM, p. 4 : Et comment aucunes devotes creatures pensans en la vierge Marie et considerant contemplativement les grans joies qu'elle pouoit avoir durans les sains misteres qui furent en l'Annunciacion, en la Nativité, en l'Ascencion Jhesu Crist et autres, qu'ilz ont mises en [.XV.] joies, ou nom et pour l'onneur desquelles pluseurs bons catholiques ont fait pluseurs belles oraisons et devotes d'icelle saincte vierge Marie, moy aussy, pensant et considerant le fait de mariage (...), ay advisé que en mariage a $[. \mathrm{XV}$.$] serimonies (...).$

4) Monstrelet, Chronique, p. 101 : Durant lesdictes trêves cessera et fera le Roy nostre dit très redoubté et souverain seigneur, cesser toute guerre et voie de fait entre lui et ses royaumes, pays et subgectz et alyés, ainsy que dict est.

Dans cette perspective, en effet, le premier exemple ressortit encore à l'emploi verbal, mais n'en est pas moins déjà potentiellement engagé dans le processus de grammaticalisation en ce sens qu'il présente réunies les conditions préalables à la mise en œuvre du processus. Un stade supplémentaire est franchi dans l'exemple suivant (2), qui tient à l'absence d'accord de durant avec les trieuwes, absence d'accord compensée en un sens par la postposition attendue du participe à son sujet. On peut dès lors tenir que le processus de formation est ici supérieurement engagé que dans le cas précédent. En (3), en revanche, c'est l'antéposition du participe qui est compensée par l'accord effectué avec le sujet (les sains misteres). Ces deux seuils constituent ainsi des seuils intermédiaires, qu'il ne nous semble pas véritablement possible, sauf à privilégier l'un des deux critères au détriment de l'autre, d'ordonner l'un par rapport à l'autre sur le vecteur. Enfin, dans le dernier cas, l'ensemble des critères (antéposition et absence d'accord) définissant le seuil maximal de grammaticalisation est présent. Cet exemple est d'autant plus intéressant qu'il semble déjà, si on le place en contraste avec l'exemple (1), issu de la même œuvre, témoigner d'une distribution complémentaire entre l'emploi verbal et l'emploi prépositionnel de la forme participiale. 
Le principe d'un continuum tel que le suggère $\mathrm{Cl}$. Buridant permet donc bien d'unifier l'interprétation de ces différentes constructions dans le cadre d'un changement en cours comme autant de seuils (initiaux, intermédiaires et finaux) menant à l'aboutissement de celui-ci. Il ne resterait plus alors qu'à postuler une diversification progressive des régimes prépositionnels, et, notamment, la possibilité d'un régime construit autour d'un verbe conjugué.

\section{Une phase de « prépositionnalisation $»^{6}$ ?}

L'existence d'un stade proprement prépositionnel ne va pourtant pas sans poser quelques difficultés, tenant aussi bien à l'empirie linguistique qu'à la théorie qui en sous-tend le postulat. Suivant la première, en effet, le premier problème qui se pose tient aux datations relatives des prépositions et des locutions. D’une façon générale, ces dernières apparaissent entre la fin de l'ancien français et le début du moyen français :

Une chose est certaine : si les locutions modernes pendant que et durant que s'ébauchent vers la fin du XIII e siècle et au début du XIVe siècle, elles n'entreront définitivement dans l'usage qu'au $\mathrm{XV}^{\mathrm{e}}$ siècle et plus sûrement encore au XVIe siècle. [Imbs 1956 : 304]

À tout le moins P. Imbs suppose-t-il une mise en place plus progressive de l'usage de durant que, et, plus généralement, des conjonctions à base participiale, en affirmant, dans la «conclusion générale» de son étude, que «l'heure de pendant que et de durant que approche (les tournures participes et prépositives sont en place), mais elle n'a guère encore sonné » (Ibid.: 556). Or, pour certaines de ces formations, l'apparition de la locution est, sinon tout à fait antérieure à celle de la préposition, du moins relativement contemporaine. Ainsi, dans le cas de durant, dont la première attestation est, pour l'emploi prépositionnel, attribuée aux Coutumes de Philippe de Beaumanoir (cf. Littré, et, à sa suite, P. Imbs (1956: 303)). L'exemple donné est généralement le suivant :

5) Beaumanoir 1, p. 176 : Durant le mariage li chevaliers acheta un fief et en fist homage au conte.

Par la suite, C. Marchello-Nizia affirme que «durant que devient conjonction au XIVe siècle » (1997 : 370). Or, T. Arnavielle relève l'exemple suivant, assez nettement antérieur, puisque ce texte est généralement daté de 1258, soit un quart de siècle avant la rédaction du Coutumier de Clermont:

\footnotetext{
${ }^{6}$ Nous entendons ici par «prépositionnalisation » l'acquisition, par un verbe, des propriétés qui en font une préposition. Nous préférons ce terme à celui de grammaticalisation - dont il pourrait être un avatar - en ce qu'il ne préjuge précisément pas du processus mis en jeu.
} 
6) Novare, Mémoires, p. 43, \LXXII : Et durant que l'on traitoit la pais, l'on manda querre Phelippe.

Encore convient-il de noter que l'édition consultée (celle de Kohler, réalisée sans consultation du manuscrit), autant que l'histoire complexe du manuscrit, ne permettent pas de tenir cet exemple pour absolument assuré. Et il semble, à consulter les variantes, que le manuscrit portait à l'origine devant que, ce qui serait sans doute plus en adéquation avec le fort usage des diverses valeurs de ce terme que l'on peut par ailleurs constater. On peut encore ajouter à cela le fait que la BFM ne donne pour première attestation de cette locution conjonctive que la forme durant ce que ${ }^{7}$ :

7) Monstrelet, Chroniques, p. 44 : Le Roy n'entent riens debvoir à monditseigneur de La Trimoulle, et envoie, le Roy, en la chambre des comptes pour sçavoir tout ce qu'il a eu de lui contre raison durant ce qu'il a esté contre luy, en sa compaignie, et depuis.

Plus probant peut-être est le cas de moyennant que. Les datations proposées par le TLFi (et non infirmées par nos corpus) sont en effet de 1219 pour la locution conjonctive, à partir de l'exemple suivant :

8) Charte de 1219: Moyennant que icelle eglise voelle a ce entendre, (cit. dans Godefroy, Complément-2 du Dictionnaire. (in Cart. de Cysoing, p. 104)).

mais seulement 1377 pour la préposition, dont nous avons trouvé dans le DMF une occurrence à peu près contemporaine :

9) Oresme (Nicole), Le Livre du Ciel et du Monde (1370), II, 15, p. 440 : Et la terre pure a puissance de engendrer chaleur moiennant tel mouvement et telle violence.

De fait, les datations relatives n'autorisent pas à affirmer que la prépositionnalisation soit une phase nécessaire au sein de la genèse des locutions participiales.

En outre, le recours à un stade prépositionnel engage une conception spécifique des locutions conjonctives, selon laquelle elles ne possèdent de réalité que sur le plan discursif. En d'autres termes, les locutions conjonctives ne seraient que la coalescence discursive d'une préposition et de l'un des régimes possibles, un régime en Que-P, substituant au parenthétisage de (10) le parenthétisage de (11) :

10) Cnn, p. 173: (Durant que ces accointances se faisoient), la viande fut apportée, et s'assiet après le maistre d'ostel chacun en sa place.

11) Cnn, p. 173 : (Durant (que ces accointances se faisoient)), la viande fut apportée, et s'assiet après le maistre d'ostel chacun en sa place.

\footnotetext{
7 Pour une étude plus précise de la présence de l'élément $c e$ dans ce type de locutions, voir Verjans (2009 : 514-521). Plus généralement, voir Combettes (2005, 2006 \& 2007).
} 
Il n'est pas possible de discuter ici des détails d'une telle conception ${ }^{8}$. On se bornera toutefois à remarquer qu'elle ne vaut d'abord que pour les cas où le premier élément est de nature prépositionnelle et exclut ainsi les formations plus complexes comme cependant que ou nonobstant que.

Une alternative a cependant été proposée par P. Imbs, selon qui la modification profonde instaurée par l'apparition de ces locutions tient au fait que la subordonnée assume désormais le rôle de sujet :

[L]'emploi de ces locutions constitue un renversement total du système traditionnel de présentation de l'idée subordonnée. Les subordonnées temporelles étaient jusque-là considérées comme de simples extensions de l'adverbe ou du complément circonstanciel de temps qui les introduisait : ce morphème introducteur servait comme de charnière entre la principale et la subordonnée. Viennent les morphèmes participes et tout change : de simple extension, la subordonnée devient sujet du verbe introducteur. Mais la fonction sujet est la position qu'une proposition subordonnée à le plus de peine à prendre. (...) [C]oncevoir un agent ou un point de départ sous la forme d'un événement, c'est se livrer à une transposition audacieuse dont les noms d'action fournissaient un modèle insuffisant. [Imbs 1956 : 305]

Suivant cette analyse, les locutions participiales émergeraient en quelque sorte directement des constructions participiales. La modification en cause ne tiendrait plus alors à la prépositionnalisation de la forme verbale elle-même, mais à la modification de la nature de ses arguments. Et si une telle analyse n'est validée par aucune occurrence dans laquelle la proposition sujet serait antéposée à la forme verbale, elle semble correspondre à des configurations dans lesquelles un participe accordé construit un régime propositionnel. Ainsi de l'exemple suivant :

12) Monstrelet, Chronique, p. 64 : En après, yceulx deffendans, considérans qu'ilz ne se povoient longuement tenir, et aussy qu'ilz n'avoient point grand espérance d'avoir aulcun secours, firent traictié avec les commis dudit conte d'Estampes, moyenant et par tel sy, qu'ilz se départiroient de là en emportant trestous leurs biens avec eulx.

Quoiqu'un tel exemple ne permette pas véritablement de trancher entre une interprétation verbale ou une interprétation déjà conjonctive, il n'en témoigne pas

\footnotetext{
${ }^{8}$ On rappellera simplement que la version la plus élaborée de cette conception est due à P. Le Goffic (cf. en particulier 1993a et b). Entre autres mérites, cette version prend en compte la diversité compositionnelle des locutions conjonctives et rend caduque un certain nombre d'arguments traditionnellement avancés contre elle. Elle peine cependant à expliquer des cas comme puisque ou tandis que, pourtant centraux dans l'empirie linguistique, et qui ne peuvent être tenus pour résiduels qu'au regard de la théorie.
} 
moins d'une structure favorable à un changement de type réanalyse, ne présupposant alors pas de passage par une phase de prépositionnalisation. C'est pourquoi l'examen d'une hypothèse alternative apparaît nécessaire.

\section{Vers un modèle analogique}

Nous avons pu apercevoir, au cours des considérations précédentes, quelques difficultés tenant tant aux critères retenus - et notamment le cas de l'accord - qu'à la modélisation elle-même du processus. En particulier, la phase de prépositionnalisation, si elle peut sembler légitime dans le cas de bon nombre des locutions ainsi formées, ne saurait rendre compte de l'entier de la catégorie et les problèmes de datation relative rencontrés en chemin invitent à davantage de prudence. En définitive, les phénomènes invoqués pour expliquer l'apparition de ces locutions conjonctives ne valent véritablement que comme des conditions préalables, mais laissent dans l'ombre l'ultime étape de cette formation : le passage de la préposition à la locution. Enfin, comme nous allons le voir, ils ne parviennent pas à rendre compte de l'ensemble de ces formations, et il reste alors à identifier la nature du ou des changement(s) en cause dans la genèse de ces locutions. Or, la productivité de ce type de réalisations, constituant, selon les termes d'A. Bertin, « un paradigme ouvert» (2003 : 268), laisse penser que c'est à un moyen systématique - voire typologique, étant donné le caractère 'européen' du phénomène - que l'on a affaire, moyen systématique qu'il est alors possible d'appréhender en s'inspirant de la théorie cosérienne.

Il est possible de recourir à l'analogie telle qu'elle est conçue dans cette théorie. Selon celle-ci, en effet, et eu égard à la tripartition norme - système - type ${ }^{9}$ (Coseriu 1952) sur laquelle elle se fonde, le mécanisme analogique doit être tenu pour une règle créatrice de formations nouvelles :

L'analogie est, en effet, un changement dans la "norme », mais non dans le "système », puisqu'elle est, au contraire, une "création systématique », une réalisation d'une possibilité du système. [Coseriu 1973 (2007) : VII, \ 1.1.2]

Dès lors, le mécanisme de l'analogie peut être conçu comme la mise en œuvre d'un moyen systématique par lequel se trouve actualisée une possibilité du système jusqu'alors demeurée à l'état de simple virtualité.

\footnotetext{
9 « La couche de la norme correspond à la réalisation «normale » d'une technique linguistique dans le discours : elle englobe tout ce qui, dans une langue, même sans être objectivement fonctionnel, et traditionnel et « usuel » (...). Le système, par contre, c'est la couche des oppositions distinctives dans l'expression et dans le contenu, c'est-à-dire l'ensemble des fonctions qui constituent une langue. Et le type linguistique, c'est la couche de l'analogie structurale fonctionnelle entre les différentes sections d'un système, la couche de l'unité supérieure des fonctions et des procédés d'une langue; par exemple, l'unité essentielle des fonctions et des procédés utilisés dans le domaine du nom et dans le domaine du verbe, dans la morphologie du mot et dans la morphologie de la phrase, etc. C'est dans ce sens que le type constitue le niveau le plus haut d'une technique linguistique : il correspond aux principes de structuration d'une langue et représente sa cohérence et son unité fonctionnelle, sousjacentes à la variété et diversité des fonctions et des procédés spécifiques au niveau du système. » [Coseriu 1987: 53-54] Voir, également, Coseriu (1952).
} 
On peut alors avancer l'existence d'un patron morphosyntaxique propre aux locutions conjonctives et formalisables comme suit :

$$
\mathrm{X}+(\mathrm{ce} / \ldots)+k^{w}
$$

Un tel patron, qui vise à représenter la forme abstraite des locutions conjonctives, doit être tenu pour une règle systématique dont l'application permet la création des locutions conjonctives, au moins en français, mais bien plus vraisemblablement, et moyennant quelques spécifications supplémentaires, dans l'ensemble des langues romanes ${ }^{10}$. Nous préciserons simplement que l'élément $\mathrm{X}$ représente l'ensemble des éléments (de nature morphémiques ou syntagmatiques) susceptibles d'intervenir dans la composition d'une locution conjonctive. Ainsi, le statut préposi-tionnel acquis par les formes participiales en a fait des candidats privilégiés à la saturation de cette position et, partant, à la constitution d'une nouvelle série de locutions conjonctives.

Mais le recours à un tel modèle présente surtout l'avantage d'unifier les explications apportées à des formations comme cependant que, nonobstant que, non contrestant que mais également pourvu que. Ces dernières relèvent, en effet, d'une composition morphologique différente, sinon plus complexe, que les autres et présentent en outre des particularités d'emplois qui ne s'accordent pas avec le principe d'une phase de prépositionnalisation. Ainsi, des locutions telles que cependant que ou pourvu que sont fondées à partir d'une base sans aucune attestation prépositionnelle ${ }^{11}$.

Dans le cas de nonobstant que - ainsi que, dans une moindre mesure, non contrestant que - qui connaît des emplois prépositionnels, le phénomène est quelque peu différent. Comme l'a souligné O. Soutet :

Le caractère tardif et rare des emplois qui témoignent d'un accord grammatical avec l'élément qui suit - lequel, sauf erreur, ne s'observe qu'avec non obstant - interdit de supposer qu'il y ait eut un stade du type «ablatif absolu ». [Soutet $1990: 161-162$ ]

On soulignera d'abord le fait que le "caractère tardif et rare des emplois qui témoignent d'un accord avec l'élément qui suit » n'a guère de quoi surprendre, puisque les constructions participiales absolues se développent, elles aussi, assez tardivement, c'est-à-dire, comme nous l'avons vu, au cours du moyen français et, plus encore, de l'époque de la Renaissance. Et si l'on tient compte du fait que les agents nominaux, ainsi que le remarquait T. Arnavielle, sont majoritairement antéposés, la rareté de l'accord se trouve, sur ce point aussi, en quelque sorte justifiée par ce que l'on pourrait appeler une forme d'iconicité syntagmatique, i.e. une correspondance sémiologique avec l'ordre des constituants. Il faut y ajouter la prédominance, au moins pour l'ancien français et les premiers temps du moyen français, de la progression à thème constant, qui contribue à faire du syntagme participial le marqueur d'une continuité référentielle. Il demeure donc tout à fait clair que l'absence d'accord, toutes proportions gardées, a favorisé la réinterprétation prépositionnelle de certains participes en constructions

\footnotetext{
10 Une nouvelle fois, nous nous permettons de renvoyer à notre thèse (Verjans 2009 : 271-374).

11 Il n'est d'ailleurs pas inintéressant de remarquer que la locution pendant que apparaît bien après la locution cependant que, puisque celle-ci est datée de la première moitié du XIVe siècle tandis que celle-là est attestée à partir de la seconde moitié du XVe siècle.
} 
absolues. Celle-ci ne suffit cependant pas à rendre compte de la formation de nonobstant que, qui provient, selon toute vraisemblance, d'une innovation introduite dans la langue des juristes à partir des modèles latins, et s'approche en cela d'un processus tel que l'emprunt. Autrement dit, il ne paraît pas que nous ayons affaire, à chaque fois, à une formation strictement graduelle, ou, à tout le moins, qui soit observable dans son déroulement.

Dès lors, le recours à une analogie de type systématique comme ultime phénomène de changement permet aussi bien de résoudre le problème posé par la dernière étape que d'unifier les différents processus à l'œuvre dans la genèse de ces locutions. Il demeure néanmoins clair qu'une étape analogique n'exclut pas l'ensemble des changements évoqués précédemment, mais, au contraire, les conserve au titre de conditions préalables, c'est-à-dire ayant favorisé l'aptitude de tel ou tel élément à saturer la position X du patron morphosyntaxique. L'analogie permet alors de rendre compte du passage de la forme participiale, qu'elle soit ou non déjà prépositionnelle, à la locution conjonctive.

\section{Conclusion}

Cette étude, encore préliminaire à de nombreux égards, avait pour enjeu d'interroger la relation entre les prépositions et les locutions conjonctives issues des formes participiales. Ces formations, principalement apparues au cours du moyen français, s'inscrivent dans le cadre d'un renouvellement plus général des processus de liaison de la phrase. Il s'agissait alors de proposer une hypothèse susceptible d'expliquer l'étape ultime de la formation des locutions participiales, ordinairement appréhendées comme une diversification des régimes prépositionnels. C'est là que le recours à l'analogie cosérienne offre une solution en permettant, à partir d'un patron morphosyntaxique, de justifier l'actualisation de la locution participiale.

Plusieurs points restent cependant à approfondir ou, plus simplement, à développer pour achever de valider cette hypothèse. En particulier, il conviendrait de prendre plus spécifiquement en compte le niveau sémantique mis en jeu dans ces évolutions et d'étendre l'analyse à chacune des locutions relevant de ce modèle. D'autre part, cette hypothèse devra être évaluée dans une perspective typologique dans la mesure où nombre de langues, romanes ou non, connaissent des formations de ce type. On peut citer, à titre d'exemple ${ }^{12}$ :

Espagnol : conforme que, dado que, en vista que, puesto que, salvo que, según que.

Catalan : donat que, llevat que, posat que, salvant que, tret que, vist que.

Portuguais : dado que, não obstante, posto que, suposto que, visto que.

Italien : ammesso che, considerato che, dato che, posto che, supposto che, visto che. durante che, nonostante che.

Anglais : considering that, outaken that, provided that, notwithstanding, providing that, conditioned that, during that, except(ing) that, assuming that, given that.

C'est en examinant l'ensemble de ces locutions qu'il sera possible de préciser la nature analogique du changement en jeu dans la genèse de ces formations.

12 Ces exemples sont pris, pour la plupart, chez B. Kortmann (1997a : 292-294 et 1997b : 37-53) ainsi que parmi nos propres corpus. 


\section{CORPUS}

\section{Bases de données}

BFM : Base de Français Médiéval, [en ligne]. Lyon: UMR ICAR/ENS-LSH, 2006, $<$ http://bfm.ens-lsh.fr $>$.

BHC : Corpus de la Littérature Médiévale en langue d'oïl des origines à la fin du XVe siècle, sous la direction de Claude Blum, Honoré Champion.

DMF : Dictionnaire du Moyen Français. ATILF / Nancy Université - CNRS. Site internet : http://www.atilf.fr/dmf.

\section{Dictionnaires}

Godefroy, F., 1881, Dictionnaire de l'ancienne langue française et de tous ses dialectes du IX au XVe siècle, 10 vol., Paris, F. Vieweg.

Greimas, A. J., [1979] 1997, Dictionnaire de l'ancien français. Le Moyen Âge, Paris, Larousse «Trésors du français».

TOBler, A. \& LommatzsCH, E., 1915, Altfranzösisches Wörterbuch, Vols. 1-10, Berlin et Wiesbaden: Franz Steiner Verlag. Vol. 11 et seq. H.H. Christmann. 1989, Stuttgart: Franz Steiner Verlag (Abr. T\&L).

\section{Abréviations}

[Beaumanoir 1] : c. 1283 - Philippe de Beaumanoir, Coutumes de Beauvaisis, tome 1.

[Cnn] : c. 1456-1467 - Anonyme, Les Cent nouvelles nouvelles.

[Froissart, 1] : c. 1369-1400 - Jean Froissart, Chroniques, livre premier.

[Monstrelet, Chronique] : 1441 - Enguerrand de Monstrelet, Chronique.

[Novare, Mémoires] : c. 1250 - Philippe de Novare, Mémoires.

[Oresme, Livre du Ciel et du Monde] : c. 1377 - Nicole Oresme, Le Livre du ciel et du monde.

[QJM] : vers 1400 - Les.XV. joies de mariage.

\section{RÉFÉRENCES BIBLIOGRAPHIQUES}

ANDRIEUX-REIX, N. \& BAUMGARTNER, E., 1983, Systèmes morphologiques de l'ancien français : le verbe, Bordeaux, Sobodi.

ARNAVIELLE, T. , 1997, Le morphème - ant : unité et diversité. Étude historique et théorique, Louvain, Peeters.

BAT-ZEEV SHYLDKROT, H., 1993, « Sur le rapport temporel-causal dans les subordonnées. Le cas de en attendant que - attendu que », La subordination in A. Boone (éd). Travaux de Linguistique, 27, p. 113-123. 
BERTIN, A., 2001, «Maintenant: un cas de grammaticalisation ?» in La linguistique diachronique : grammaticalisation et sémantique du prototype, in De Mulder W. \& Vanderheyden A. (éds) Langue Française, 130 (mai 2001), p. 42-64.

BERTIN, A., 2003, «Les connecteurs de cause dans l'histoire du français - contradictions du changement linguistique », Verbum, XXV-3, p. 263-276.

BURIDANT, Cl., 2000, Grammaire nouvelle de l'ancien français, Paris, SEDES.

COMBETTES, B., 2003, «L'évolution de la forme en -ant: aspects syntaxiques et textuels », Langages, 149 , p. 6-24.

COMBETTES, B., 2005, «La grammaticalisation des locutions conjonctives en français: l'opposition que/ce que », Colloque « Coordination et subordination », pré-texte (texte paru dans Faits de langue, 14, 2006, p. 95-106.

CombetTes, B., 2006, «La formation des locutions conjonctives temporelles : le cas de dès que », in Moline, E., Stosic, D. \& Vetters, C. (éds) Les connecteurs temporels du français, Cahiers Chronos, 15, p. 1-19.

COMBETTES, B., 2007, «La formation des locutions conjonctives temporelles : l'opposition que / ce que en moyen français", in P. Le Goffic (éd.) Les mots en qu- du français, Lexique, Villeneuve d'Ascq, Presses Universitaires du Septentrion, p. 47-67.

COSERIU, E., 1952, "Sistema, norma y habla », Revista de la Faculdad de Humanidades y de Ciencias, VI, 9, p. 113-181.

COSERIU, E., [1973] 2007, Sincronía, diacronía e historia. El problema del cambio lingüístico, Madrid, Gredos. Trad. de T. Verjans Synchronie, diachronie et histoire. Le problème du changement linguistique, édition Texto!, [en ligne].

COSERIU, E., 1987, "Le latin vulgaire et le type linguistique roman », in J. Herman (éd.) Latin vulgaire - latin tardif, Acte du $1^{e r}$ Colloque international sur le latin vulgaire et tardif (Pécs, 2 - 5 septembre 1985), Tübingen, p. 53-64.

FAGARD, B. \& De Mulder, W., 2007, «La formation de prépositions complexes : grammaticalisation ou lexicalisation. », in Prévost, S. \& Fagard, B. (éds) Grammaticalisation et lexicalisation : la formation d'expressions complexes, Langue française, 156, p. 9-29.

Fragonard, M.-M. \& KotLer, E., 1994, Introduction à la langue du XVTe siècle, Paris, Nathan-128.

IMBS, P., 1956, Les propositions temporelles en ancien français. La détermination du moment, Publications de la Faculté des Lettres de Strasbourg.

LE GOFFIC, P., 1993a, Grammaire de la phrase française, Paris, Hachette.

LE GOFFIC, P., 1993b, «Les subordonnées circonstancielles et le classement formel des subordonnées », in Claude Guimier (éd), 1001 circonstants, Caen, Presses Universitaires de Caen, p. 69-102.

LE GOFFIC , P., 2001, «Sur les sources et le développement de la subordination dans le langage : l'exemple de l'indo-européen », in Dupuy-Engelhardt, H., Palma, S. \& 
Tyvaert, J.-E. (éds), Les phrases dans les textes, les sons et les mots pour les dire, les connecteurs du discours, l'opposition verbo-nominale en acte, Actes des journées scientifiques 2000, Recherches en Linguistique et Psychologie cognitive ${ }^{\circ}{ }^{16}$, Presses Universitaires de Reims, p. 25-56.

LERCH, E. (1924-1935), Historische französische Syntax, Leipzig, O. R. Reisland.

KÖNIG, E. \& van der AUWERA, J. , 1990, « Adverbial participles, gerunds, and absolute constructions », in J. Bechert, G. Bernini \& Cl. Buridant (éds) Toward a Typology of European Languages, Berlin-New York, Mouton de Gruyter "Empirical Approaches to Language Typology", 8, p. 337-355.

KÖNIG, E. \& KORTMANN, B., 1991, «On the reanalysis of verb as prepositions » in Gisa Rauh (ed.), Approaches to Prepositions, Tübingen, Gunter narr Verlag, p. 109-125.

KÖnig, E. \& Kortmann, B., 1992, «Categorial Reanalysis: The Case of Deverbal Prepositions », Linguistics, 30, p. 671-697.

Kortmann, B., 1992, «Reanalysis Completed and in Progress: Participles as Source of Prepositions and Conjunctions », in G. Kellermann \& M.D. Morrissey (eds), Diachrony within Synchrony : Language History and Cognition, Frankfurt, M. Lang, p. 429-453.

Kortmann, B., 1997a, Adverbial Subordination. A Typology and History of Adverbial Subordinators Based on European Languages, Berlin/New York, Mouton de Gruyter (Empirical Approaches to Language Typology).

Kortmann, B., 1997b, A cross-linguistic dictionary of adverbial subordinators, Munich, Lincom Europa, http://www.anglistik.uni-freiburg.de/institut/lskortmann/

MARCHELlO-NIZIA, Chr. (1997), La langue française au XIVee et XVe siècles, Paris, Nathan.

MEYER-LÜBKE, W., 1974, Grammaire des langues romanes (trad. Auguste et Georges Doutrepont), 4. Vol.

Soutet, O., 1990, La concession en français des origines an XVTe siècle. Problèmes généraux. Les tours prépositionnels, Genève, Droz.

VERJANS, Th., 2009, Essai de systématique diachronique : genèse des conjonctions en français (IX - XVII siècle), thèse de doctorat n.r., Université Paris-Sorbonne.

VerJans, Th., sous presse, «Les locutions participiales : une catégorie marginale? », Actes du colloque international "Morphologie, syntaxe et sémantique des subordonnants", C. Bodelot, H. Gruet-Skrabalova, Fr. Trouilleux (éds), Clermont-Ferrand, Cabiers $d u$ LRL, n ${ }^{\circ} 5$.

ZINK, G., 1993, L'Ancien français, Paris, PUF (QSJ ?) (1ère éd. 1987). 